\title{
REPRESENTATION OF SOLUTIONS OF A TWO-DIMENSIONAL SYSTEM OF DIFFERENCE EQUATIONS
}

\author{
Y. HALIM, A. KHELIFA, AND M. BERKAL
}

Received 23 January, 2020

\begin{abstract}
In this paper we give a representation formula for the general solution to the following two-dimensional system of difference equations

$$
x_{n+1}=\frac{y_{n-1} x_{n-2}}{y_{n}\left(a+b y_{n-1} x_{n-2}\right)}, \quad y_{n+1}=\frac{x_{n-1} y_{n-2}}{x_{n}\left(a+b x_{n-1} y_{n-2}\right)}, \quad n \in \mathbb{N}_{0}
$$

where parameters $a, b$ and initial values $x_{-2}, x_{-1}, x_{0}, y_{-2}, y_{-1}, y_{0}$ are real numbers. We also give some theoretical explanations related to the representation.
\end{abstract}

2010 Mathematics Subject Classification: 39A10; 40A05

Keywords: system of difference equations, general solution, representation of solutions

\section{INTRODUCTION}

Solvability of difference equations and system of difference equations has attracted considerable interest recently (see, for example [1-19], and the related references therein).

The following four systems of difference equations

$$
x_{n+1}=\frac{y_{n-1} x_{n-2}}{y_{n}\left( \pm 1 \pm y_{n-1} x_{n-2}\right)}, \quad y_{n+1}=\frac{x_{n-1} y_{n-2}}{x_{n}\left( \pm 1 \pm x_{n-1} y_{n-2}\right)}, \quad n \in \mathbb{N}_{0}
$$

have been studied in [5], where some closed-form formulas for their solutions are given in terms of the initial values $x_{-2}, x_{-1}, x_{0}, y_{-2}, y_{-1}, y_{0}$. The closed-form formulas are given and proved by using the method of induction.

In this work we give an alternative proof in order to explain theoretically the results presented in [5], which were established through a mere application of the induction principle.

Here we consider the following extension of the systems in (1.1)

$$
x_{n+1}=\frac{y_{n-1} x_{n-2}}{y_{n}\left(a+b y_{n-1} x_{n-2}\right)}, \quad y_{n+1}=\frac{x_{n-1} y_{n-2}}{x_{n}\left(a+b x_{n-1} y_{n-2}\right)}, \quad n \in \mathbb{N}_{0}
$$

This work was supported by "Directorate general for Scientific Research and Technological Development (DGRSDT), Algeria". 
where parameters $a, b$ and initial values $x_{-2}, x_{-1}, x_{0}, y_{-2}, y_{-1}, y_{0}$ are real numbers.

Our objective is to show that system (1.2) is solvable by finding its closed-form formulas through an analytical approach, and to show that all the closed-form formulas obtained in [5] easily follow from the ones in our present paper.

\section{MAIN RESULTS}

Assume that $\left\{x_{n}, y_{n}\right\}_{n \geq-2}$ is a well-defined solution to system (1.2).

Let

$$
u_{n}=x_{n} y_{n-1}, \quad v_{n}=y_{n} x_{n-1},
$$

for $n \geq-1$. Then system (1.2) can be written as

$$
u_{n+1}=\frac{v_{n-1}}{a+b v_{n-1}}, \quad v_{n+1}=\frac{u_{n-1}}{a+b u_{n-1}}, \quad n \in \mathbb{N}_{0} .
$$

To give a closed-form for the well-defined solutions of the system (2.2), we consider the system of two difference equations of first order

$$
u_{n+1}=\frac{v_{n}}{a+b v_{n}}, \quad v_{n+1}=\frac{u_{n}}{a+b u_{n}}, \quad n \in \mathbb{N}_{0} .
$$

The system (2.3) can be written as the following equation

$$
u_{n+1}=\frac{u_{n-1}}{a^{2}+b(a+1) u_{n-1}} .
$$

Let

$$
u_{n}^{(j)}=u_{2 n-j}, \quad n \in \mathbb{N}, j \in\{0,1\} .
$$

Using notation (2.5), we can write (2.4) as

$$
u_{n+1}^{(j)}=\frac{u_{n}^{(j)}}{a^{2}+b(a+1) u_{n}^{(j)}},
$$

where $j \in\{0,1\}$.

Equation (2.6) can be reduced to the equation :

$$
\mathcal{H}_{n+1}=\frac{\left(a^{2}+1\right) \mathcal{H}_{n}-a^{2}}{\mathcal{H}_{n}},
$$

by using the change of variable

$$
u_{n}^{(j)}=\frac{1}{b(a+1)}\left(\mathcal{H}_{n}-a^{2}\right) .
$$

Now we consider the difference equation (2.7) with the initial value $\mathcal{H}_{0}$ is non zero real number.

Through an analytical approach, we put

$$
\mathcal{H}_{n}=\frac{k_{n}}{k_{n-1}} .
$$


Then equation (2.7) becomes

$$
k_{n+1}-\left(a^{2}+1\right) k_{n}-a^{2} k_{n-1}=0, \quad n \in \mathbb{N}_{0} .
$$

Case $a^{2} \neq 1$ :

Let $\left\{k_{n}\right\}_{n>-1}$ be the solution to equation (2.10) such that $k_{0}$ and $k_{-1} \in \mathbb{R}$. The zeros of the characteristic polynomial $P(\lambda)=\lambda^{2}-\left(a^{2}+1\right) \lambda+a^{2}$ are $\lambda_{1}=a^{2}$ and $\lambda_{2}=1$. Then the general solution to equation (2.10) can be written in the following form

$$
k_{n}=c_{1}+c_{2} a^{2 n}
$$

Using the initial values $k_{0}$ and $k_{-1}$ with some calculations, we get

$$
\begin{aligned}
& c_{1}=\frac{k_{0}-k_{-1} a^{2}}{1-a^{2}}, \\
& c_{2}=\frac{a^{2}\left(k_{-1}-k_{0}\right)}{1-a^{2}} .
\end{aligned}
$$

So the general solution of equation (2.10) is

$$
k_{n}=\frac{1}{1-a^{2}}\left[k_{0}\left(1-a^{2(n+1)}\right)-a^{2} k_{-1}\left(1-a^{2 n}\right)\right] .
$$

From all above mentioned we see that the following theorem holds.

Theorem 1. Let $\left\{\mathcal{H}_{n}\right\}_{n \geq 0}$ be a well-defined solution to the equation (2.7). Then, for $n=2,3, \ldots$,

$$
\mathcal{H}_{n}=\frac{A\left(1-a^{2 n}\right)-\mathcal{H}_{0}\left(1-a^{2(n+1)}\right)}{a^{2}\left(1-a^{2(n-1)}\right)-\mathcal{H}_{0}\left(1-a^{2 n}\right)} .
$$

Then, from (2.8) we see that

$$
u_{n}^{(j)}=\frac{1}{b(a+1)}\left(\mathcal{H}_{n}-a^{2}\right)=\frac{u_{0}^{(j)}}{a^{2 n}+b(a+1) u_{0}^{(j)} \sum_{r=0}^{n-1} a^{2 r}},
$$

for each $j \in\{0,1\}$.

Case $a^{2}=1$ :

Then equation (2.7) becomes

$$
k_{n+1}-2 k_{n}-k_{n-1}=0, \quad n \in \mathbb{N}_{0},
$$

Let $\left\{k_{n}\right\}_{n \geq-1}$ be the solution to equation (2.13) such that $k_{0}$ and $k_{-1} \in \mathbb{R}$. The zero of the characteristic polynomial $P(\lambda)=(\lambda-1)^{2}$ is $\lambda_{1}=1$. Then the general solution to equation (2.13) can be written in the following form

$$
k_{n}=c_{1}+c_{2} n \text {. }
$$


Using the initial values $k_{0}$ and $k_{-1}$ with some calculations we get

$$
\begin{aligned}
& c_{1}=k_{0} \\
& c_{2}=k_{0}-k_{-1} .
\end{aligned}
$$

So the general solution of equation (2.13) is

$$
k_{n}=k_{0}(n+1)+k_{-1} n .
$$

From all above mentioned we see that the following theorem holds.

Theorem 2. Let $\left\{\mathcal{H}_{n}\right\}_{n \geq 0}$ be a well-defined solution to the equation (2.7). Then, for $n=2,3, \ldots$,

$$
\mathcal{H}_{n}=\frac{n-\mathcal{H}_{0}(n+1)}{(n-1)-\mathcal{H}_{0} n} .
$$

Then, from (2.8) we see that

$$
u_{n}^{(j)}=\frac{u_{0}^{(j)}}{1+b(a+1) u_{0}^{(j)} n}
$$

where $j \in\{0,1\}$.

From all above mentioned with using (2.5) we see that the following corollary holds.

Corollary 1. Let $\left\{u_{n}\right\}_{n \geq-1}$ be a well-defined solution to the equation (2.4). Then

$$
\begin{aligned}
\text { if } a^{2} \neq 1: & u_{2 n-j}=\frac{u_{-j}}{a^{2 n}+b(a+1) u_{-j} \sum_{r=0}^{n-1} a^{2 r}}, \\
\text { if } a^{2}=1: & u_{2 n-j}=\frac{u_{-j}}{1+b(a+1) u_{-j} n},
\end{aligned}
$$

where $j \in\{0,1\}$.

Corollary 2. Let $\left\{u_{n}, v_{n}\right\}_{n \geq 0}$ be a well-defined solution to the system (2.3). Then if $a^{2} \neq 1$ :

$$
\begin{array}{ll}
u_{2 n}=\frac{u_{0}}{a^{2 n}+b(a+1) u_{0} \sum_{r=0}^{n-1} a^{2 r}}, & u_{2 n+1}=\frac{v_{0}}{a^{2 n+1}+b v_{0}\left(a \sum_{r=0}^{n-1} a^{2 r}+\sum_{r=0}^{n} a^{2 r}\right)}, \\
v_{2 n}=\frac{v_{0}}{a^{2 n}+b(a+1) v_{0} \sum_{r=0}^{n-1} a^{2 r}}, & v_{2 n+1}=\frac{a^{2 n+1}+b u_{0}\left(a \sum_{r=0}^{n-1} a^{2 r}+\sum_{r=0}^{n} a^{2 r}\right)}{} .
\end{array}
$$

if $a^{2}=1$ :

$$
u_{2 n}=\frac{u_{0}}{1+b(a+1) n u_{0}}, \quad u_{2 n+1}=\frac{v_{0}}{a+b((a+1) n+1) v_{0}},
$$




$$
v_{2 n}=\frac{v_{0}}{1+b(a+1) n v_{0}}, \quad v_{2 n+1}=\frac{u_{0}}{a+b((a+1) n+1) u_{0}},
$$

where $n \in \mathbb{N}_{0}$.

Proof. Let $\left\{u_{n}, v_{n}\right\}_{n>0}$ be a solution of system (2.3), so $\left\{u_{n}\right\}_{n \geq-1}$ is a solution of equation (2.6). Then, if $a^{2} \neq 1$, let

$$
u_{2 n-1}=\frac{u_{-1}}{a^{2 n}+b(a+1) u_{-1} \sum_{r=0}^{n-1} a^{2 r}},
$$

and

$$
v_{0}=\frac{u_{-1}}{a+b u_{-1}},
$$

so

$$
\begin{aligned}
u_{2 n+1}= & \frac{u_{-1}}{a^{2(n+1)}+b(a+1)\left(\sum_{r=0}^{n} a^{2 r}\right) u_{-1}} \\
= & \frac{u_{-1}}{a^{2 n+1}\left(a+b u_{-1}\right)+b\left(a \sum_{r=0}^{n-1} a^{2 r}+\sum_{r=0}^{n} a^{2 r}\right) u_{-1}} \\
= & \frac{v_{0}}{a^{2 n+1}+b\left(a\left(\sum_{i=0}^{n-1} a^{2 r}\right)+\left(\sum_{i=0}^{n} a^{2 r}\right)\right) v_{0}} .
\end{aligned}
$$

if $a^{2}=1$, let

$$
u_{2 n-1}=\frac{u_{-1}}{1+b(a+1) n u_{-1}}
$$

and

$$
v_{0}=\frac{u_{-1}}{a+b u_{-1}},
$$

so

$$
\begin{aligned}
u_{2 n+1} & =\frac{u_{-1}}{1+b(a+1)(n+1) u_{-1}}=\frac{u_{-1}}{a^{2}+b(a+1)(n+1) u_{-1}} \\
& =\frac{u_{-1}}{a\left(a+b u_{-1}\right)+b((a+1) n+1) u_{-1}}=\frac{v_{0}}{a+b((a+1) n+1) v_{0}} .
\end{aligned}
$$

In the same way, after some calculation and use that

$$
v_{n}=\frac{u_{n-1}}{a+b u_{n-1}}
$$


we obtain, if $a^{2} \neq 1$, that

$$
v_{2 n}=\frac{v_{0}}{a^{2 n}+b(a+1) v_{0} \sum_{r=0}^{n-1} a^{2 r}}, \quad v_{2 n+1}=\frac{u_{0}}{a^{2 n+1}+b u_{0}\left(a \sum_{r=0}^{n-1} a^{2 r}+\sum_{r=0}^{n} a^{2 r}\right)},
$$

and, if $a^{2}=1$, that

$$
v_{2 n}=\frac{v_{0}}{1+b(a+1) n v_{0}}, \quad v_{2 n+1}=\frac{u_{0}}{a+b((a+1) n+1) u_{0}} .
$$

Go back now to the system (2.2), we using an appropriate transformation reducing this system to the system of first-order difference equations (2.3).

The initial values with the smallest indexes are $u_{-k}$ and $v_{-k}$. By using (2.2) with $n=0$, we obtain the values of $u_{1}$ and $v_{1}$ as follows

$$
u_{1}=\frac{v_{-1}}{a+b v_{-1}}, \quad v_{1}=\frac{u_{-1}}{a+b u_{-1}} .
$$

After known the values of $u_{1}$ and $v_{1}$, by using (2.2) with $n=2$ we get the values of $u_{3}$ and $v_{3}$. We have

$$
u_{3}=\frac{v_{1}}{a+b v_{1}}, \quad v_{3}=\frac{u_{1}}{a+b u_{1}} .
$$

The values of $u_{3}$ and $v_{3}$, by using (2.2) with $n=4$, leads us to obtain the values of $u_{5}$ and $v_{5}$. We have

$$
\begin{gathered}
u_{5}=\frac{v_{3}}{a+b v_{3}}, \quad v_{5}=\frac{u_{3}}{a+b u_{3}} . \\
u_{2 m+1}=\frac{v_{2 m-1}}{a+b v_{2 m-1}}, \quad v_{2 m+1}=\frac{u_{2 m-1}}{a+b u_{2 m-1}} .
\end{gathered}
$$

In the same way, it is shown that the initial values $u_{-i}$ and $v_{-i}$, for a fixed $i \in\{0,1\}$, determine all the values of the sequences $\left(u_{2(m+1)-i}\right)_{m}$ and $\left(v_{2(m+1)-i}\right)_{m}$. Also we have

$$
u_{2(m+1)-i}=\frac{v_{2 m-i}}{a+b v_{2 m-i}}, \quad v_{2(m+1)-i}=\frac{u_{2 m-i}}{a+b u_{2 m-i}} .
$$

Let

$$
u_{n}^{(i)}=u_{2 n-i}, \quad v_{n}^{(i)}=v_{2 n-i} .
$$

Using notation (2.18), we can write (2.2) as

$$
u_{n+1}^{(i)}=\frac{v_{n}^{(i)}}{a+b v_{n}^{(i)}}, \quad v_{n+1}^{(i)}=\frac{u_{n}^{(i)}}{a+b u_{n}^{(i)}} .
$$

From all above mentioned we see that the following theorem holds. 
Theorem 3. Let $\left\{u_{n}, v_{n}\right\}_{n \geq-1}$ be a well-defined solution to the system (2.2). Then, for $n=2,3, \ldots$, if $a^{2} \neq 1$ :

$$
\begin{aligned}
& u_{4 n-1}=\frac{u_{-1}}{a^{2 n}+b(a+1) \sum_{r=0}^{n-1} a^{2 r} u_{-1}}, \\
& v_{4 n-1}=\frac{v_{-1}}{a^{2 n}+b(a+1) \sum_{r=0}^{n-1} a^{2 r} v_{-1}}, \\
& u_{4 n}=\frac{u_{0}}{a^{2 n}+b(a+1) \sum_{r=0}^{n-1} a^{2 r} u_{0}}, \\
& u_{4 n+1}=\frac{v_{-1}}{a^{2 n+1}+b\left(a \sum_{r=0}^{n-1} a^{2 r}+\sum_{r=0}^{n} a^{2 r}\right) v_{-1}}, \\
& u_{4 n+2}=\frac{v_{0}}{a^{2 n+1}+b\left(a \sum_{r=0}^{n-1} a^{2 r}+\sum_{r=0}^{n} a^{2 r}\right) v_{0}}, \\
& v_{4 n}=\frac{v_{0}}{a^{2 n}+b(a+1) \sum_{r=0}^{n-1} a^{2 r} v_{0}} \\
& v_{4 n+1}=\frac{u_{-1}}{a^{2 n+1}+b\left(a \sum_{r=0}^{n-1} a^{2 r}+\sum_{r=0}^{n} a^{2 r}\right) u_{-1}}, \\
& v_{4 n+2}=\frac{u_{0}}{a^{2 n+1}+b\left(a \sum_{r=0}^{n-1} a^{2 r}+\sum_{r=0}^{n} a^{2 r}\right) u_{0}} \text {. } \\
& u_{4 n-1}=\frac{u_{-1}}{1+b(a+1) n u_{-1}}, \\
& u_{4 n}=\frac{u_{0}}{1+b(a+1) n u_{0}}, \\
& u_{4 n+1}=\frac{v_{-1}}{a+b((a+1) n+1) v_{-1}}, \\
& v_{4 n-1}=\frac{v_{-1}}{1+b(a+1) n v_{-1}}, \\
& u_{4 n+2}=\frac{v_{0}}{a+b((a+1) n+1) v_{0}}, \\
& v_{4 n}=\frac{v_{0}}{1+b(a+1) n v_{0}}, \\
& v_{4 n+1}=\frac{u_{-1}}{a+b((a+1) n+1) u_{-1}}, \\
& v_{4 n+2}=\frac{u_{0}}{a+b((a+1) n+1) u_{0}} .
\end{aligned}
$$

where $n \in \mathbb{N}_{0}$.

From (2.1) we have

$$
\begin{aligned}
x_{n} & =\frac{u_{n}}{y_{n-1}}, \\
y_{n} & =\frac{v_{n}}{x_{n-1}} .
\end{aligned}
$$

Using (2.20) in (2.19), we obtain

$$
x_{4 n}=\frac{u_{4 n} u_{4 n-2}}{v_{4 n-1} v_{4 n-3}} x_{4 n-4} .
$$

Using (2.19) in (2.20), we obtain

$$
y_{4 n}=\frac{v_{4 n} v_{4 n-2}}{u_{4 n-1} u_{4 n-3}} y_{4 n-4} .
$$


For $n \in \mathbb{N}$, multiplying the equalities which are obtained form (2.21) and (2.22) from 1 to $n$, respectively, it follows that

$$
\begin{aligned}
& x_{4 n}=x_{0} \prod_{i=0}^{n-1}\left(\frac{u_{4 i} u_{4 i-2}}{v_{4 i-1} v_{4 i-3}}\right), \\
& y_{4 n}=y_{0} \prod_{i=0}^{n-1}\left(\frac{v_{4 i} v_{4 i-2}}{u_{4 i-1} u_{4 i-3}}\right) .
\end{aligned}
$$

Using the equalities (2.23) and (2.24) in (2.19) and (2.20), we obtain

$$
x_{4 n-1}=\frac{v_{6 n}}{y_{6 n}}=\frac{v_{4 n}}{y_{0}} \prod_{i=0}^{n-1}\left(\frac{u_{4 i-1} u_{4 i-3}}{v_{4 i} v_{4 i-2}}\right) .
$$

We have

$$
y_{4 n-1}=\frac{u_{4 n}}{x_{4 n}}=\frac{u_{4 n}}{x_{0}} \prod_{i=0}^{n-1}\left(\frac{v_{4 i-1} v_{4 i-3}}{u_{4 i} u_{4 i-2}}\right) .
$$

Using the equalities (2.25) and (2.26) in (2.19) and (2.20), we obtain

$$
x_{4 n-2}=\frac{v_{4 n-1}}{y_{4 n-1}}=x_{0} \frac{v_{4 n-1}}{u_{4 n}} \prod_{i=0}^{n-1}\left(\frac{u_{4 i} u_{4 i-2}}{v_{4 i-1} v_{4 i-3}}\right),
$$

and

$$
y_{4 n-2}=\frac{u_{4 n-1}}{x_{4 n-1}}=y_{0} \frac{u_{4 n-1}}{v_{4 n}} \prod_{i=0}^{n-1}\left(\frac{v_{4 i} v_{4 i-2}}{u_{4 i-1} u_{4 i-3}}\right) .
$$

Using the equalities (2.27) and (2.28) in (2.19) and (2.20), we obtain

$$
x_{4 n+1}=\frac{u_{4 n+1}}{y_{4 n}}=\frac{u_{4 n+1}}{y_{0}} \prod_{i=0}^{n-1}\left(\frac{u_{4 i-1} u_{4 i-3}}{v_{4 i} v_{4 i-2}}\right),
$$

and

$$
y_{4 n+1}=\frac{v_{4 n+1}}{x_{4 n}}=\frac{v_{4 n-1}}{x_{0}} \prod_{i=0}^{n-1}\left(\frac{v_{4 i-1} v_{4 i-3}}{u_{4 i} u_{4 i-2}}\right) .
$$

Using Theorem (3) we get

$$
\text { if } a^{2} \neq 1 \text { : }
$$

$$
\begin{aligned}
& u_{4 n-3}=\frac{v_{-1}}{a^{2 n-1}+b\left(a \sum_{r=0}^{n-2} a^{2 r}+\sum_{r=0}^{n-1} a^{2 r}\right) v_{-1}}, \quad v_{4 n-3}=\frac{u_{-1}}{v^{2 n-1}+b\left(a \sum_{r=0}^{n-2} a^{2 r}+\sum_{r=0}^{n-1} a^{2 r}\right) u_{-1}}, \\
& u_{4 n-2}=\frac{v_{0}}{a^{2 n-1}+b\left(a \sum_{r=0}^{n-2} a^{2 r}+\sum_{r=0}^{n-1} a^{2 r}\right) v_{0}}, \quad v_{4 n-2}=\frac{u^{2 n-1}+b\left(a \sum_{r=0}^{n-2} a^{2 r}+\sum_{r=0}^{n-1} a^{2 r}\right) u_{0}}{a_{r=0}},
\end{aligned}
$$




$$
\begin{aligned}
u_{4 n-1}= & \frac{u_{-1}}{a^{2 n}+b(a+1) \sum_{r=0}^{n-1} a^{2 r} u_{-1}} \\
u_{4 n}= & \frac{u_{0}}{a^{2 n}+b(a+1) \sum_{r=0}^{n-1} a^{2 r} u_{0}}
\end{aligned}
$$

$$
\begin{aligned}
v_{4 n-1}= & \frac{v_{-1}}{a^{2 n}+b(a+1) \sum_{r=0}^{n-1} a^{2 r} v_{-1}}, \\
v_{4 n}= & \frac{v_{0}}{a^{2 n}+b(a+1) \sum_{r=0}^{n-1} a^{2 r} v_{0}} .
\end{aligned}
$$

if $a^{2}=1$ :

$$
\begin{aligned}
u_{4 n-3} & =\frac{v_{-1}}{a+b((a+1) n-a) v_{-1}}, & v_{4 n-3} & =\frac{u_{-1}}{a+b((a+1) n-a) u_{-1}}, \\
u_{4 n-2} & =\frac{v_{0}}{a+b((a+1) n-a) v_{0}}, & v_{4 n-2} & =\frac{u_{0}}{a+b((a+1) n-a) u_{0}}, \\
u_{4 n-1} & =\frac{u_{-1}}{1+b(a+1) n u_{-1}}, & v_{4 n-1} & =\frac{v_{-1}}{1+b(a+1) n v_{-1}}, \\
u_{4 n} & =\frac{u_{0}}{1+b(a+1) n u_{0}}, & v_{4 n} & =\frac{v_{0}}{1+b(a+1) n v_{0}} .
\end{aligned}
$$

From all above mentioned and

$$
u_{-1}=x_{-1} y_{-2}, \quad u_{0}=x_{0} y_{-1}, \quad v_{-1}=y_{-1} x_{-2}, \quad v_{0}=y_{0} x_{-1} .
$$

we see that the following result holds.

Theorem 4. Let $\left\{x_{n}, y_{n}\right\}_{n \geq-2}$ be a well-defined solution to the system (1.2). Then, for $n=0,1,2,3, \ldots$, if $a^{2} \neq 1$ :

$$
\begin{aligned}
x_{4 n-2}= & \prod_{i=0}^{n-1}\left(\frac{\left(a^{2 n}+b(a+1) \sum_{r=0}^{n-1} a^{2 r} y_{-1} x_{-2}\right)\left(a^{2 n-1}+b\left(a \sum_{r=0}^{n-2} a^{2 r}+\sum_{r=0}^{n-1} a^{2 r}\right) x_{-1} y_{-2}\right)}{\left(a^{2 n}+b(a+1) \sum_{r=0}^{i-1} a^{2 r} x_{0} y_{-1}\right)\left(a^{2 i-1}+b\left(a \sum_{r=0}^{i-2} a^{2 r}+\sum_{r=0}^{i-1} a^{2 r}\right) y_{0} x_{-1}\right)}\right) \\
& \times \frac{x_{0}^{n} y_{0}^{n}}{y_{-2}^{n} x_{-2}^{n-1}\left(\frac{a^{2 n}+b(a+1) \sum_{r=0}^{n-1} a^{2 r} x_{0} y_{-1}}{a^{2 n}+b(a+1) \sum_{r=0}^{n-1} a^{2 r} y_{-1} x_{-2}}\right),} \\
x_{4 n-1}= & \prod_{i=0}^{n-1}\left(\frac{\left(a^{2 n}+b(a+1) \sum_{r=0}^{i-1} a^{2 r} y_{0} x_{-1}\right)\left(a^{2 n-1}+b\left(a \sum_{r=0}^{i-2} a^{2 r}+\sum_{r=0}^{i-1} a^{2 r}\right) x_{0} y_{-1}\right)}{\left(a^{2 n}+b(a+1) \sum_{r=0}^{i-1} a^{2 r} x_{-1} y_{-2}\right)\left(a^{2 n-1}+b\left(a \sum_{r=0}^{i-2} a^{2 r}+\sum_{r=0}^{i-1} a^{2 r}\right) y_{-1} x_{-2}\right)}\right)
\end{aligned}
$$




$$
\begin{aligned}
& \times \frac{x_{-1} y_{-2}^{n} x_{-2}^{n}}{x_{0}^{n} y_{0}^{n}}\left(\frac{1}{a^{2 n}+b(a+1) \sum_{r=0}^{n-1} a^{2 r} y_{0} x_{-1}}\right) \text {, } \\
& x_{4 n}=\frac{x_{0}^{n+1} y_{0}^{n}}{y_{-2}^{n} x_{-2}^{n}} \\
& \prod_{i=0}^{n-1}\left(\frac{\left(a^{2 n}+b(a+1) \sum_{r=0}^{n-1} a^{2 r} y_{-1} x_{-2}\right)\left(a^{2 n-1}+b\left(a \sum_{r=0}^{n-2} a^{2 r}+\sum_{r=0}^{n-1} a^{2 r}\right) x_{-1} y_{-2}\right)}{\left(a^{2 n}+b(a+1) \sum_{r=0}^{i-1} a^{2 r} x_{0} y_{-1}\right)\left(a^{2 i-1}+b\left(a \sum_{r=0}^{i-2} a^{2 r}+\sum_{r=0}^{i-1} a^{2 r}\right) y_{0} x_{-1}\right)}\right), \\
& x_{4 n+1}=\prod_{i=0}^{n-1}\left(\frac{\left(a^{2 n}+b(a+1) \sum_{r=0}^{i-1} a^{2 r} y_{0} x_{-1}\right)\left(a^{2 n-1}+b\left(a \sum_{r=0}^{i-2} a^{2 r}+\sum_{r=0}^{i-1} a^{2 r}\right) x_{0} y_{-1}\right)}{\left(a^{2 n}+b(a+1) \sum_{r=0}^{i-1} a^{2 r} x_{-1} y_{-2}\right)\left(a^{2 n-1}+b\left(a \sum_{r=0}^{i-2} a^{2 r}+\sum_{r=0}^{i-1} a^{2 r}\right) y_{-1} x_{-2}\right)}\right) \\
& \times \frac{y_{-1} y_{-2}^{n} x_{-2}^{n+1}}{x_{0}^{n} y_{0}^{n+1}}\left(\frac{1}{a^{2 n+1}+b\left(a \sum_{r=0}^{n-1} a^{2 r}+\sum_{r=0}^{n} a^{2 r}\right) y_{-1} x_{-2}}\right) \text {, } \\
& y_{4 n-2}=\prod_{i=0}^{n-1}\left(\frac{\left(a^{2 n}+b(a+1) \sum_{r=0}^{n-1} a^{2 r} y_{-2} x_{-1}\right)\left(a^{2 n-1}+b\left(a \sum_{r=0}^{n-2} a^{2 r}+\sum_{r=0}^{n-1} a^{2 r}\right) x_{-2} y_{-1}\right)}{\left(a^{2 n}+b(a+1) \sum_{r=0}^{i-1} a^{2 r} x_{-1} y_{0}\right)\left(a^{2 i-1}+b\left(a \sum_{r=0}^{i-2} a^{2 r}+\sum_{r=0}^{i-1} a^{2 r}\right) y_{-1} x_{0}\right)}\right) \\
& \times \frac{x_{0}^{n} y_{0}^{n}}{y_{-2}^{n-1} x_{-2}^{n}}\left(\frac{a^{2 n}+b(a+1) \sum_{r=0}^{n-1} a^{2 r} x_{-1} y_{0}}{a^{2 n}+b(a+1) \sum_{r=0}^{n-1} a^{2 r} y_{-2} x_{-1}}\right) \text {, } \\
& y_{4 n-1}=\prod_{i=0}^{n-1}\left(\frac{\left(a^{2 n}+b(a+1) \sum_{r=0}^{i-1} a^{2 r} y_{-1} x_{0}\right)\left(a^{2 n-1}+b\left(a \sum_{r=0}^{i-2} a^{2 r}+\sum_{r=0}^{i-1} a^{2 r}\right) x_{-1} y_{0}\right)}{\left(a^{2 n}+b(a+1) \sum_{r=0}^{i-1} a^{2 r} x_{-2} y_{-1}\right)\left(a^{2 n-1}+b\left(a \sum_{r=0}^{i-2} a^{2 r}+\sum_{r=0}^{i-1} a^{2 r}\right) y_{-2} x_{-1}\right)}\right) \\
& \times \frac{y_{-1} y_{-2}^{n} x_{-2}^{n}}{x_{0}^{n} y_{0}^{n}}\left(\frac{1}{a^{2 n}+b(a+1) \sum_{r=0}^{n-1} a^{2 r} y_{-1} x_{0}}\right) \text {, }
\end{aligned}
$$




$$
\begin{aligned}
y_{4 n}= & \frac{x_{0}^{n} y_{0}^{n+1}}{y_{-2}^{n} x_{-2}^{n}} \\
& \prod_{i=0}^{n-1}\left(\frac{\left(a^{2 n}+b(a+1) \sum_{r=0}^{n-1} a^{2 r} y_{-2} x_{-1}\right)\left(a^{2 n-1}+b\left(a \sum_{r=0}^{n-2} a^{2 r}+\sum_{r=0}^{n-1} a^{2 r}\right) x_{-2} y_{-1}\right)}{\left(a^{2 n}+b(a+1) \sum_{r=0}^{i-1} a^{2 r} x_{-1} y_{0}\right)\left(a^{2 i-1}+b\left(a \sum_{r=0}^{i-2} a^{2 r}+\sum_{r=0}^{i-1} a^{2 r}\right) y_{-1} x_{0}\right)}\right), \\
y_{4 n+1}= & \prod_{i=0}^{n-1}\left(\frac{\left(a^{2 n}+b(a+1) \sum_{r=0}^{i-1} a^{2 r} y_{-1} x_{0}\right)\left(a^{2 n-1}+b\left(a \sum_{r=0}^{i-2} a^{2 r}+\sum_{r=0}^{i-1} a^{2 r}\right) x_{-1} y_{0}\right)}{\left(a^{2 n}+b(a+1) \sum_{r=0}^{i-1} a^{2 r} x_{-2} y_{-1}\right)\left(a^{2 n-1}+b\left(a \sum_{r=0}^{i-2} a^{2 r}+\sum_{r=0}^{i-1} a^{2 r}\right) y_{-2} x_{-1}\right)}\right) \\
& \times \frac{x_{-1} y_{-2}^{n+1} x_{-2}^{n}}{x_{0}^{n+1} y_{0}^{n}}\left(\frac{1}{a^{2 n+1}+b\left(a \sum_{r=0}^{n-1} a^{2 r}+\sum_{r=0}^{n} a^{2 r}\right) y_{-2} x_{-1}}\right)
\end{aligned}
$$

if $a^{2}=1$ :

$$
\begin{aligned}
x_{4 n-2}= & \prod_{i=0}^{n-1}\left(\frac{\left(1+b(a+1) i y_{-1} x_{-2}\right)\left(a+b((a+1) i-a) x_{-1} y_{-2}\right)}{\left(1+b(a+1) i x_{0} y_{-1}\right)\left(a+b((a+1) i-a) y_{0} x_{-1}\right)}\right) . \\
& \times \frac{x_{0}^{n} y_{0}^{n}}{y_{-2}^{n} x_{-2}^{n-1}}\left(\frac{1+b(a+1) n x_{0} y_{-1}}{1+b(a+1) n x_{-2} y_{-1}}\right), \\
x_{4 n-1}= & \prod_{i=0}^{n-1}\left(\frac{\left(1+b(a+1) i y_{0} x_{-1}\right)\left(a+b((a+1) i-a) x_{0} y_{-1}\right)}{\left(1+b(a+1) i x_{-1} y_{-2}\right)\left(a+b((a+1) i-a) y_{-1} x_{-2}\right)}\right) \\
& \times \frac{x_{-1} y_{-2}^{n} x_{-2}^{n}}{x_{0}^{n} y_{0}^{n}}\left(\frac{1}{1+b(a+1) n y_{0} x_{-1}}\right), \\
x_{4 n}= & \frac{x_{0}^{n+1} y_{0}^{n}}{y_{-2}^{n} x_{-2}^{n}} \prod_{i=0}^{n-1}\left(\frac{\left(1+b(a+1) i y_{-1} x_{-2}\right)\left(a+b((a+1) i-a) x_{-1} y_{-2}\right)}{\left(1+b(a+1) i x_{0} y_{-1}\right)\left(a+b((a+1) i-a) y_{0} x_{-1}\right)}\right), \\
x_{4 n+1}= & \prod_{i=0}^{n-1}\left(\frac{\left(1+b(a+1) i y_{0} x_{-1}\right)\left(a+b((a+1) i-a) x_{0} y_{-1}\right)}{\left(1+b(a+1) i x_{-1} y_{-2}\right)\left(a+b((a+1) i-a) y_{-1} x_{-2}\right)}\right) \\
& \times \frac{y_{-1}}{x_{0}^{n} y_{0}^{n+1}}\left(\frac{y_{-2}^{n} x_{-2}^{n+1}}{a+b((a+1) n+1) y_{-1} x_{-2}}\right), \\
y_{4 n-2}= & \frac{x_{0}^{n} y_{0}^{n}}{y_{-2}^{n-1} x_{-2}^{n}}\left(\frac{1+b(a+1) n x_{-1} y_{0}}{1+b(a+1) n y_{-2} x_{-1}}\right)
\end{aligned}
$$




$$
\begin{aligned}
& \prod_{i=0}^{n-1}\left(\frac{\left(1+b(a+1) i y_{-2} x_{-1}\right)\left(a+b((a+1) i-a) x_{-2} y_{-1}\right)}{\left(1+b(a+1) i x_{-1} y_{0}\right)\left(a+b((a+1) i-a) y_{-1} x_{0}\right)}\right), \\
y_{4 n-1}= & \frac{y_{-1} y_{-2}^{n} x_{-2}^{n}}{x_{0}^{n} y_{0}^{n}}\left(\frac{1}{1+b(a+1) n y_{-1} x_{0}}\right) \\
& \prod_{i=0}^{n-1}\left(\frac{\left(1+b(a+1) i y_{-1} x_{0}\right)\left(a+b((a+1) i-a) x_{-1} y_{0}\right)}{\left(1+b(a+1) i x_{-2} y_{-1}\right)\left(a+b((a+1) i-a) y_{-2} x_{-1}\right)}\right), \\
y_{4 n}= & \frac{x_{0}^{n} y_{0}^{n+1}}{y_{-2}^{n} x_{-2}^{n}} \prod_{i=0}^{n-1}\left(\frac{\left(1+b(a+1) i y_{-2} x_{-1}\right)\left(a+b((a+1) i-a) x_{-2} y_{-1}\right)}{\left(1+b(a+1) i x_{-1} y_{0}\right)\left(a+b((a+1) i-a) y_{-1} x_{0}\right)}\right), \\
y_{4 n+1}= & \prod_{i=0}^{n-1}\left(\frac{\left(1+b(a+1) i y_{-1} x_{0}\right)\left(a+b((a+1) i-a) x_{-1} y_{0}\right)}{\left(1+b(a+1) i x_{-2} y_{-1}\right)\left(a+b((a+1) i-a) y_{-2} x_{-1}\right)}\right) \\
& \times \frac{x_{-1}}{x_{0}^{n+1} y_{0}^{n}}\left(\frac{y_{-2}^{n+1} x_{-2}^{n}}{a+b((a+1) n+1) y_{-2} x_{-1}}\right) .
\end{aligned}
$$

\section{SOME APPLICATIONS}

As some applications we show how are obtained closed-form formulas for solutions to the systems in (1.1), which were presented in [5].

First result proved in [5] is the following.

Corollary 3. Let $\left\{x_{n}, y_{n}\right\}_{n \geq-2}$ be a well-defined solution to the following system

$$
x_{n+1}=\frac{y_{n-1} x_{n-2}}{y_{n}\left(1+y_{n-1} x_{n-2}\right)}, \quad y_{n+1}=\frac{x_{n-1} y_{n-2}}{x_{n}\left(1+x_{n-1} y_{n-2}\right)}, \quad n \in \mathbb{N}_{0} .
$$

Then

$$
\begin{aligned}
x_{4 n-2} & =\frac{x_{0}^{n} y_{0}^{n}}{y_{-2}^{n} x_{-2}^{n-1}}\left(\frac{1+2 n x_{0} y_{-1}}{1+2 n x_{-2} y_{-1}}\right) \prod_{i=0}^{n-1}\left(\frac{\left(1+2 i y_{-1} x_{-2}\right)\left(1+(2 i-1) x_{-1} y_{-2}\right)}{\left(1+2 i x_{0} y_{-1}\right)\left(1+(2 i-a) y_{0} x_{-1}\right)}\right), \\
x_{4 n-1} & =\frac{x_{-1} y_{-2}^{n} x_{-2}^{n}}{x_{0}^{n} y_{0}^{n}}\left(\frac{1}{1+2 n y_{0} x_{-1}}\right) \prod_{i=0}^{n-1}\left(\frac{\left(1+2 i y_{0} x_{-1}\right)\left(1+(2 i-a) x_{0} y_{-1}\right)}{\left(1+2 i x_{-1} y_{-2}\right)\left(1+(2 i-1) y_{-1} x_{-2}\right)}\right), \\
x_{4 n} & =\frac{x_{0}^{n+1} y_{0}^{n}}{y_{-2}^{n} x_{-2}^{n}} \prod_{i=0}^{n-1}\left(\frac{\left(1+2 i y_{-1} x_{-2}\right)\left(1+(2 i-a) x_{-1} y_{-2}\right)}{\left(1+2 i x_{0} y_{-1}\right)\left(1+(2 i-a) y_{0} x_{-1}\right)}\right), \\
x_{4 n+1} & =\frac{y_{-1}}{x_{0}^{n} y_{0}^{n+1}}\left(\frac{y_{-2}^{n} x_{-2}^{n+1}}{1+(2 n+1) y_{-1} x_{-2}}\right) \prod_{i=0}^{n-1}\left(\frac{\left(1+2 i y_{0} x_{-1}\right)\left(1+\left((2 i-1) x_{0} y_{-1}\right)\right.}{\left(1+2 i x_{-1} y_{-2}\right)\left(1+(2 i-1) y_{-1} x_{-2}\right)}\right),
\end{aligned}
$$

and

$y_{4 n-2}=\frac{x_{0}^{n} y_{0}^{n}}{y_{-2}^{n-1} x_{-2}^{n}}\left(\frac{1+2 n x_{-1} y_{0}}{1+2 n y_{-2} x_{-1}}\right) \prod_{i=0}^{n-1}\left(\frac{\left(1+2 i y_{-2} x_{-1}\right)\left(1+(2 i-1) x_{-2} y_{-1}\right)}{\left(1+2 i x_{-1} y_{0}\right)\left(1+(2 i-a) y_{-1} x_{0}\right)}\right)$, 


$$
\begin{aligned}
y_{4 n-1} & =\frac{y_{-1} y_{-2}^{n} x_{-2}^{n}}{x_{0}^{n} y_{0}^{n}}\left(\frac{1}{1+2 i y_{-1} x_{0}}\right) \prod_{i=0}^{n-1}\left(\frac{\left(1+2 i y_{-1} x_{0}\right)\left(1+(2 i-1) x_{-1} y_{0}\right)}{\left(1+2 i x_{-2} y_{-1}\right)\left(1+(2 i-1) y_{-2} x_{-1}\right)}\right), \\
y_{4 n} & =\frac{x_{0}^{n} y_{0}^{n+1}}{y_{-2}^{n} x_{-2}^{n}} \prod_{i=0}^{n-1}\left(\frac{\left(1+2 i y_{-2} x_{-1}\right)\left(1+(2 i-a) x_{-2} y_{-1}\right)}{\left(1+2 i x_{-1} y_{0}\right)\left(1+(2 i-1) y_{-1} x_{0}\right)}\right), \\
y_{4 n+1} & =\frac{x_{-1}}{x_{0}^{n+1} y_{0}^{n}}\left(\frac{y_{-2}^{n+1} x_{-2}^{n}}{1+(2 n+1) y_{-2} x_{-1}}\right) \prod_{i=0}^{n-1}\left(\frac{\left(1+2 i y_{-1} x_{0}\right)\left(1+(2 i-1) x_{-1} y_{0}\right)}{\left(1+2 i x_{-2} y_{-1}\right)\left(1+(2 i-1) y_{-2} x_{-1}\right)}\right) .
\end{aligned}
$$

Proof. System (3.1) is obtained from system (1.2) with $a=b=1$, so by using Theorem (4) corollary (3) follows.

The following corollary is Theorem 2.2 in [5].

Corollary 4. Let $\left\{x_{n}, y_{n}\right\}_{n \geq-2}$ be a well-defined solution to the following system

$$
x_{n+1}=\frac{y_{n-1} x_{n-2}}{y_{n}\left(1-y_{n-1} x_{n-2}\right)}, \quad y_{n+1}=\frac{x_{n-1} y_{n-2}}{x_{n}\left(1-x_{n-1} y_{n-2}\right)}, \quad n \in \mathbb{N}_{0} .
$$

Then

$$
\begin{aligned}
x_{4 n-2} & =\frac{x_{0}^{n} y_{0}^{n}}{y_{-2}^{n} x_{-2}^{n-1}}\left(\frac{1-2 n x_{0} y_{-1}}{1-2 n x_{-2} y_{-1}}\right) \prod_{i=0}^{n-1}\left(\frac{\left(1-2 i y_{-1} x_{-2}\right)\left(1-(2 i-1) x_{-1} y_{-2}\right)}{\left(1-2 i x_{0} y_{-1}\right)\left(1-(2 i-1) y_{0} x_{-1}\right)}\right), \\
x_{4 n-1} & =\frac{x_{-1} y_{-2}^{n} x_{-2}^{n}}{x_{0}^{n} y_{0}^{n}}\left(\frac{1}{1-2 n y_{0} x_{-1}}\right) \prod_{i=0}^{n-1}\left(\frac{\left(1-2 i y_{0} x_{-1}\right)\left(1-(2 i-1) x_{0} y_{-1}\right)}{\left(1-2 i x_{-1} y_{-2}\right)\left(1-(2 i-1) y_{-1} x_{-2}\right)}\right), \\
x_{4 n} & =\frac{x_{0}^{n+1} y_{0}^{n}}{y_{-2}^{n} x_{-2}^{n}} \prod_{i=0}^{n-1}\left(\frac{\left(1-2 i y_{-1} x_{-2}\right)\left(1-(2 i-1) x_{-1} y_{-2}\right)}{\left(1-2 i x_{0} y_{-1}\right)\left(1-(2 i-1) y_{0} x_{-1}\right)}\right), \\
x_{4 n+1} & =\frac{y_{-1}}{x_{0}^{n} y_{0}^{n+1}}\left(\frac{y_{-2}^{n} x_{-2}^{n+1}}{1-(2 n+1) y_{-1} x_{-2}}\right) \prod_{i=0}^{n-1}\left(\frac{\left(1-2 i y_{0} x_{-1}\right)\left(1-(2 i-1) x_{0} y_{-1}\right)}{\left(1-i x_{-1} y_{-2}\right)\left(1-(2 i-1) y_{-1} x_{-2}\right)}\right), \\
y_{4 n-2} & =\frac{x_{0}^{n} y_{0}^{n}}{y_{-2}^{n-1} x_{-2}^{n}}\left(\frac{1-2 n x_{-1} y_{0}}{1-2 n y_{-2} x_{-1}}\right) \prod_{i=0}^{n-1}\left(\frac{\left(1-2 i y_{-2} x_{-1}\right)\left(1-(2 i-1) x_{-2} y_{-1}\right)}{\left(1-2 i x_{-1} y_{0}\right)\left(1-(2 i-1) y_{-1} x_{0}\right)}\right), \\
y_{4 n-1} & =\frac{y_{-1} y_{-2}^{n} x_{-2}^{n}}{x_{0}^{n} y_{0}^{n}}\left(\frac{1}{1-2 n y_{-1} x_{0}}\right) \prod_{i=0}^{n-1}\left(\frac{\left(1-2 i y_{-1} x_{0}\right)\left(1-(2 i-1) x_{-1} y_{0}\right)}{\left(1-2 i x_{-2} y_{-1}\right)\left(1-(2 i-1) y_{-2} x_{-1}\right)}\right), \\
y_{4 n}= & \frac{x_{0}^{n} y_{0}^{n+1}}{y_{-2}^{n} x_{-2}^{n}} \prod_{i=0}^{n-1}\left(\frac{\left(1-2 i y_{-2} x_{-1}\right)\left(1-(2 i-1) x_{-2} y_{-1}\right)}{\left(1-2 i x_{-1} y_{0}\right)\left(1-(2 i-1) y_{-1} x_{0}\right)}\right), \\
y_{4 n+1} & =\frac{x_{-1}}{x_{0}^{n+1} y_{0}^{n}}\left(\frac{y_{-2}^{n+1} x_{-2}^{n}}{1-(2 n+1) y_{-2} x_{-1}}\right) \prod_{i=0}^{n-1}\left(\frac{\left(1-2 i y_{-1} x_{0}\right)\left(1-(2 i-1) x_{-1} y_{0}\right)}{\left(1-2 i x_{-2} y_{-1}\right)\left(1-(2 i-1) y_{-2} x_{-1}\right)}\right) .
\end{aligned}
$$

Proof. System (3.2) is obtained from system (1.2) with $a=1$ and $b=-1$, so by using Theorem (4) corollary (4) follows. 
The following corollary is Theorem 5.3 in [5].

Corollary 5. Let $\left\{x_{n}, y_{n}\right\}_{n \geq-2}$ be a well-defined solution to the following system

$$
x_{n+1}=\frac{y_{n-1} x_{n-2}}{y_{n}\left(-1+y_{n-1} x_{n-2}\right)}, \quad y_{n+1}=\frac{x_{n-1} y_{n-2}}{x_{n}\left(-1+x_{n-1} y_{n-2}\right)}, \quad n \in \mathbb{N}_{0} .
$$

Then

$$
\begin{aligned}
x_{4 n-2} & =\frac{x_{0}^{n} y_{0}^{n}\left(-1+x_{-1} y_{-2}\right)^{n}}{y_{-2}^{n} x_{-2}^{n-1}\left(-1+y_{0} x_{-1}\right)^{n}}, & y_{4 n-2} & =\frac{y_{0}^{n} x_{0}^{n}\left(-1+y_{-1} x_{-2}\right)^{n}}{x_{-2}^{n} y_{-2}^{n-1}\left(-1+x_{0} y_{-1}\right)^{n}}, \\
x_{4 n-1} & =\frac{x_{-1} y_{-2}^{n} x_{-2}^{n}\left(-1+x_{0} y_{-1}\right)^{n}}{x_{0}^{n} y_{0}^{n}\left(-1+y_{-1} x_{-2}\right)^{n}}, & y_{4 n-1} & =\frac{y_{-1} x_{-2}^{n} y_{-2}^{n}\left(-1+y_{0} y_{-1}\right)^{n}}{y_{0}^{n} x_{0}^{n}\left(-1+x_{-1} y_{-2}\right)^{n}}, \\
x_{4 n} & =\frac{x_{0}^{n+1} y_{0}^{n}\left(-1+x_{-1} y_{-2}\right)^{n}}{y_{-2}^{n} x_{-2}^{n}\left(-1+y_{0} x_{-1}\right)^{n}}, & y_{4 n} & =\frac{y_{0}^{n+1} x_{0}^{n}\left(-1+y_{-1} x_{-2}\right)^{n}}{x_{-2}^{n} y_{-2}^{n}\left(-1+x_{0} y_{-1}\right)^{n}}, \\
x_{4 n+1} & =\frac{y_{-1} y_{-2}^{n} x_{-2}^{n+1}\left(-1+x_{0} y_{-1}\right)^{n}}{x_{0}^{n} y_{0}^{n+1}\left(-1+y_{-1} x_{-2}\right)^{n+1}}, & y_{4 n+1} & =\frac{x_{-1} x_{-2}^{n} y_{-2}^{n+1}\left(-1+y_{0} x_{-1}\right)^{n}}{y_{0}^{n} x_{0}^{n+1}\left(-1+x_{-1} y_{-2}\right)^{n+1}} .
\end{aligned}
$$

Proof. System (3.3) is obtained from system (1.2) with $a=1$ and $b=-1$, so by using Theorem (4) corollary (5) follows.

The following corollary is Theorem 5.4 in [5].

Corollary 6. Let $\left\{x_{n}, y_{n}\right\}_{n \geq-2}$ be a well-defined solution to the following system

$$
x_{n+1}=\frac{y_{n-1} x_{n-2}}{y_{n}\left(-1-y_{n-1} x_{n-2}\right)}, \quad y_{n+1}=\frac{x_{n-1} y_{n-2}}{x_{n}\left(-1-x_{n-1} y_{n-2}\right)}, \quad n \in \mathbb{N}_{0} .
$$

Then

$$
\begin{aligned}
x_{4 n-2} & =\frac{x_{0}^{n} y_{0}^{n}\left(-1-x_{-1} y_{-2}\right)^{n}}{y_{-2}^{n} x_{-2}^{n-1}\left(-1-y_{0} x_{-1}\right)^{n}}, & y_{4 n-2} & =\frac{y_{0}^{n} x_{0}^{n}\left(-1-y_{-1} x_{-2}\right)^{n}}{x_{-2}^{n} y_{-2}^{n-1}\left(-1-x_{0} y_{-1}\right)^{n}}, \\
x_{4 n-1} & =\frac{x_{-1} y_{-2}^{n} x_{-2}^{n}\left(-1-x_{0} y_{-1}\right)^{n}}{x_{0}^{n} y_{0}^{n}\left(-1-y_{-1} x_{-2}\right)^{n}}, & y_{4 n-1} & =\frac{y_{-1} x_{-2}^{n} y_{-2}^{n}\left(-1-y_{0} y_{-1}\right)^{n}}{y_{0}^{n} x_{0}^{n}\left(-1-x_{-1} y_{-2}\right)^{n}}, \\
x_{4 n} & =\frac{x_{0}^{n+1} y_{0}^{n}\left(-1-x_{-1} y_{-2}\right)^{n}}{y_{-2}^{n} x_{-2}^{n}\left(-1-y_{0} x_{-1}\right)^{n}}, & y_{4 n-1} & =\frac{y_{-1} x_{-2}^{n} y_{-2}^{n}\left(-1-y_{0} y_{-1}\right)^{n}}{y_{0}^{n} x_{0}^{n}\left(-1-x_{-1} y_{-2}\right)^{n}}, \\
x_{4 n+1} & =\frac{y_{-1} y_{-2}^{n} x_{-2}^{n+1}\left(-1-x_{0} y_{-1}\right)^{n}}{x_{0}^{n} y_{0}^{n+1}\left(-1-y_{-1} x_{-2}\right)^{n+1}}, & y_{4 n+1} & =\frac{x_{-1} x_{-2}^{n} y_{-2}^{n+1}\left(-1-y_{0} x_{-1}\right)^{n}}{y_{0}^{n} x_{0}^{n+1}\left(-1-x_{-1} y_{-2}\right)^{n+1}} .
\end{aligned}
$$

Proof. System (3.4) is obtained from system (1.2) with $a=b=-1$, so by using Theorem (4) corollary (6) follows. 


\section{REFERENCES}

[1] Y. Akrour, N. Touafek, and Y. Halim, "On a system of difference equations of second order solved in closed-form." Miskolc Math. Notes, vol. 20, no. 2, pp. 701-717, 2019, doi: 10.18514/MMN.2019.2923.

[2] E. M. Elsayed, "Solutions of rational difference systems of order two," Math. Comput. Modelling, vol. 55, no. 1, pp. 378-384, 2012, doi: 10.1016/j.mcm.2011.08.012.

[3] E. M. Elsayed, "Solution for systems of difference equations of rational form of order two," Comp. Appl. Math., vol. 33, no. 3, pp. 751-765, 2014, doi: 10.1007/s40314-013-0092-9.

[4] E. M. Elsayed, "On a system of two nonlinear difference equations of order two," Proc. Jangjeon Math. Soc., vol. 18, no. 3, pp. 353-368, 2015.

[5] E. M. Elsayed and T. F. Ibrahim, "Periodicity and solutions for some systems of nonlinear rational difference equations," Hacet. J. Math. Stat., vol. 44, no. 6, pp. 1361-1390, 2015, doi: 10.15672/HJMS.2015449653.

[6] Y. Halim, "Form and periodicity of solutions of some systems of higher-order difference equations," Math. Sci. Lett., vol. 5, no. 1, pp. 79-84, 2016, doi: 10.18576/ms1/050111.

[7] Y. Halim, "A system of difference equations with solutions associated to Fibonacci numbers," Int. J. Difference. Equ., vol. 11, no. 1, pp. 65-77, 2016.

[8] Y. Halim, "Global character of systems of rational difference equations," Electron. J. Math. Analysis. Appl., vol. 3, no. 1, pp. 204-214, 2018.

[9] Y. Halim and M. Bayram, "On the solutions of a higher-order difference equation in terms of generalized Fibonacci sequences,” Math. Methods Appl. Sci., vol. 39, no. 1, pp. 2974-2982, 2016, doi: 10.1002/mma.3745.

[10] Y. Halim and J. F. T. Rabago, "On the solutions of a second-order difference equation in terms of generalized Padovan sequences,” Math. Slovaca, vol. 68, no. 3, pp. 625-638, 2018, doi: 10.1515/ms-2017-0130.

[11] M. Kara and Y. Yazlik, "Solvability of a system of nonlinear difference equations of higher order," Turk. J. Math., vol. 43, no. 3, pp. 1533-1565, 2019, doi: 10.3906/mat-1902-24.

[12] M. Kara and Y. Yazlik, "On the system of difference equations $x_{n}=\frac{x_{n-2} y_{n-3}}{y_{n-1}\left(a_{n}+b_{n} x_{n-2} y_{n-3}\right)}, \quad y_{n}=$ $\frac{y_{n-2} x_{n-3}}{x_{n-1}\left(\alpha_{n}+\beta_{n} y_{n-2} x_{n-3}\right)}$, J. Math. Ext., vol. 14, no. 1, pp. 41-59, 2020.

[13] A. Khelifa, Y. Halim, and M. Berkal, "Solutions of a system of two higher-order difference equations in terms of Lucas sequence," Univers. J. Math. Appl., vol. 2, no. 4, pp. 202-211, 2019, doi: 10.32323/ujma.610399.

[14] S. Stevic, "Representation of solutions of bilinear difference equations in terms of generalized Fibonacci sequences," Electron. J. Qual. Theory Differ. Equ., no. 67, pp. 1-15, 2014, doi: 10.14232/ejqtde.2014.1.67.

[15] S. Stevic, B. Iricanin, W. Kosmala, and Z. Smarda, "Representation of solutions of a solvable nonlinear difference equation of second order," Electron. J. Qual. Theory Differ. Equ., no. 95, pp. 1-18, 2018, doi: 10.14232/ejqtde.2018.1.95.

[16] D. T. Tollu, Y. Yazlik, and N. Taskara, "On the solutions of two special types of Riccati difference equation via Fibonacci numbers," Adv. Difference Equ., vol. 174, no. 1, pp. 1-7, 2013, doi: 10.1186/1687-1847-2013-174.

[17] D. T. Tollu, Y. Yazlik, and N. Taskara, "The solutions of four Riccati difference equations associated with Fibonacci numbers," Balkan J. Math., vol. 2, no. 1, pp. 163-172, 2014.

[18] Y. Yazlik and N. Taskara, "A note on generalized $k$-Horadam sequence," Comput. Math. Appl., vol. 63, no. 1, pp. 36-41, 2012.

[19] Y. Yazlik, D. T. Tollu, and N. Taskara, "On the solutions of difference equation systems with Padovan numbers," Appl. Math., vol. 12, no. 1, pp. 15-202, 2013. 
Authors' addresses

\section{Y. Halim}

Yacine Halim, Abdelhafid Boussouf University of Mila, Department of Mathematics and Computer Science, and LMAM Laboratory, University of Mohamed Seddik Ben Yahia, Jijel, Algeria

E-mail address: halyacine@yahoo.fr

\section{A. Khelifa}

Amira Khelifa, University of Mohamed Seddik Ben Yahia, LMAM Laboratory and Department of Mathematics, Jijel, Algeria

E-mail address: amkhelifa@yahoo.com

\section{Berkal}

Massaoud Berkal, Abdelhafid Boussouf University of Mila, Department of mathematics and computer sciences, Mila, Algeria

E-mail address: berkalmessaoud@gmail.com 